\title{
Investigating the Role of Sulfides in the Space Weathering of Carbonaceous Chondrites
}

\author{
Laura Chaves $^{1}$, Michelle Thompson ${ }^{1}$ and Mark Loeffler ${ }^{2}$ \\ ${ }^{1}$ Purdue University, West Lafayette, Indiana, United States, ${ }^{2}$ Northern Arizona University, Flagstaff, \\ Arizona, United States
}

Introduction: The microstructure, chemistry, and spectral characteristics of regolith grains on the surfaces of airless bodies (e.g., Mercury, the Moon, and asteroids) are altered by solar wind irradiation and micrometeorite impacts through a process known as space weathering [1]. The microstructural and chemical characteristics of space weathering include amorphous rims, vesiculated textures, and submicroscopic Fe-bearing particles [2]. Spectrally, space weathering causes changes in the slope (i.e., reddening or bluing), reduction of reflectance (i.e., darkening) and attenuation of characteristic absorption bands [3]. Laboratory simulations and analysis of returned samples from the Moon and asteroid Itokawa have focused on space weathering signatures in silicate minerals that are the principal constituents of these collections $[4,5,6]$. However, transmission electron microscopy (TEM) analyses of samples from Itokawa identified the presence of FeS nanoparticles [10], indicating that sulfide minerals could contribute to the development of space weathering features on airless bodies. Sulfides are also important phases in more compositionally complex samples like carbonaceous chondrites which are thought to be analogs for the target asteroids of the OSIRIS-REx and Hayabusa sample return missions [9]. Despite the evidence that these phases play an important role in space weathering on asteroidal surfaces, a detailed study of their chemical and microstructural characteristics has not yet been performed. In this study, we analyzed Fesulfides from a chip of the CM2 Murchison meteorite that was subjected to simulated micrometeorite impacts. These results will be compared to natural space weathered sulfides from asteroid Itokawa.

Methods: In order to simulate micrometeorite bombardment, we subjected a dry-cut chip of the Murchison carbonaceous chondrite to pulsed-laser irradiation. The irradiation was performed using an $\mathrm{Nd}-$ YAG laser (1064 nm) with a pulse duration of 6-8 $\mathrm{ns}$, a pulse energy of $48 \mathrm{~mJ}$ and under high vacuum $\left(\sim 10^{-8}\right.$ Torr $)$ at Northern Arizona University. We used an FEI Nova Nano200 scanning electron microscopy (SEM) with an Oxford X-Max energy-dispersive X-Ray spectroscopy (EDS) detector to identify the morphological, textural and chemical changes produced on sulfide minerals after irradiation. We prepared an electron-transparent lamella from a Fe-sulfide grain using the FEI Quanta 3D FEG and a Thermo Scientific Helios G4 UX focused ion beam SEM (FIB-SEM) instrument at Purdue University. To analyze changes in the microstructure and chemistry of these samples, we used the Tecnai T20 TEM at Purdue.

Results: SEM analyses show a vesiculated texture in the meteorite matrix the presence of melt features that exhibit abundant fractures and vesicles (Fig. 1). Backscattered electron imaging (BSE) show regions with differences in contrast within the same melt that suggest a variable chemical composition. EDS chemical maps indicate that the melts are depleted in $\mathrm{Si}, \mathrm{Mg}$, and $\mathrm{O}$ but enriched in $\mathrm{Fe}$ and $\mathrm{S}$ compared to the matrix, with localized enrichments in Ni. Bright-field transmission electron microscope (BF TEM) images show the presence of a melt layer in the top of the FIB section that varies in thickness from 100 $\mathrm{nm}$ to $500 \mathrm{~nm}$ (Fig. 2). BF TEM images show the presence of vesicles in the melt layer with diameters that range from $10 \mathrm{~nm}$ to $350 \mathrm{~nm}$ and that are predominantly concentrated in the melt-grain boundary. Nanoparticles were identified in the melt and have diameters that range from $5 \mathrm{~nm}$ to $15 \mathrm{~nm}$. 
Discussion: The pulsed-laser irradiation experiments produced similar microstructural features as previous space weathering simulations and analyses of naturally space weathered samples. The melts identified on SEM analyses have a smooth appearance, fractures, and vesicles as previous laser experiments performed on Fe-sulfides [11]. Vesicles have also been identified in FeS wafers that were irradiated with $\mathrm{H}^{+}$and $\mathrm{He}^{+}$ions [12] but in this study the vesicles do not present regular shapes. Vesiculated rims have been identified in sulfides phases in particles from asteroid Itokawa [8] where the vesicles are distributed along the altered layer, however, in Murchison these vesicles are predominantly located in the intersection between the melt and the unaltered mineral grain. The nanoparticles present in the melt layer of irradiated Murchison meteorite correspond to the first nanoparticles identified in sulfide minerals. Nanoparticles embedded in the melt layer observed in the Murchison chip have a more restricted size range $(5-15 \mathrm{~nm})$ compared to nanoparticles found in agglutinates and silicate grains from lunar soils [3]. The composition of these nanoparticles will be determined by high-resolution transmission electron microscopy (HRTEM) and EDS analyses. These results will be compared to analyses of five regolith particles from asteroid Itokawa allocated to us by the Japan Aerospace Exploration Agency (JAXA).

Acknowledgments: This work was supported by NASA Laboratory Analysis of Returned Sample Grant 80NSSC19K0938.

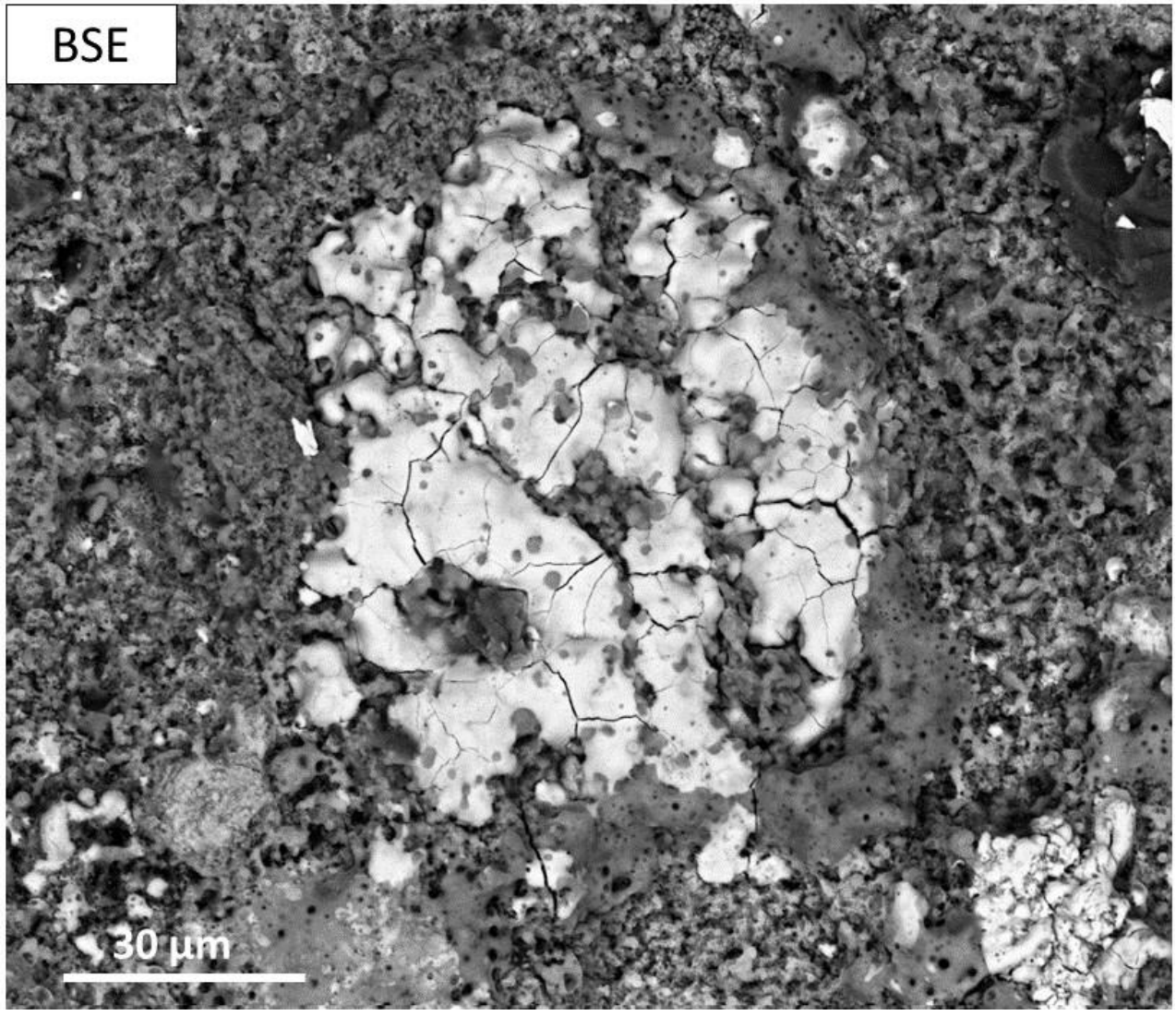

Figure 1. BSE image showing fractures, vesicles, and differences in contrast in a melt. 


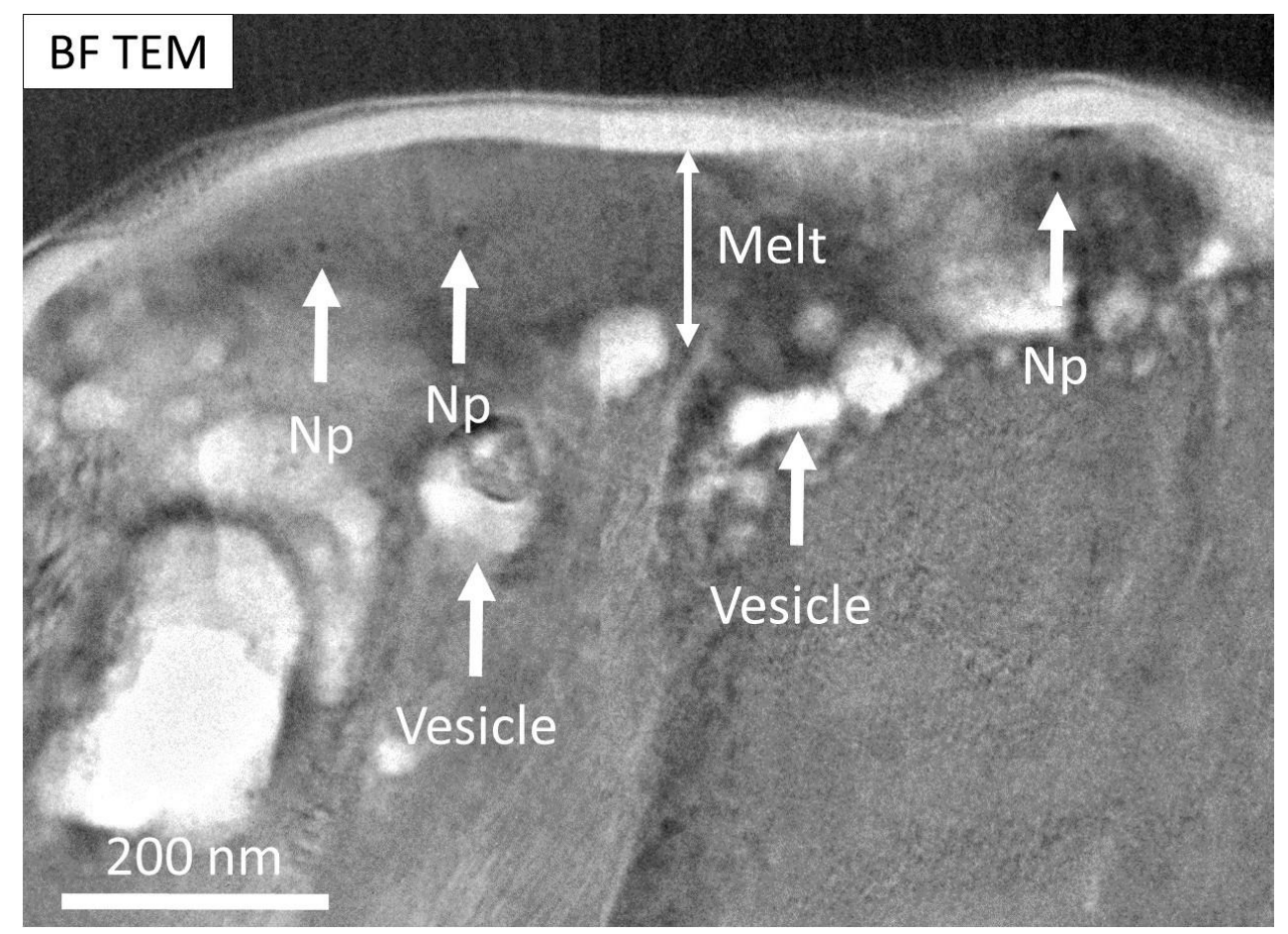

Figure 2. BF TEM image showing the melt layer, vesicles, and nanoparticles at the top of a sulfide grain.

\section{References}

[1] Hapke B. (2001) JGR 116:10039-10073.

[2] Keller L. P. et al. (1997) GCA 61: 2331-2341.

[3] Pieters C. et al. (2016) JGR 121: 1865-1884.

[4] Noguchi T. et al. (2011) Science 333:1121-1124.

[5] Thompson M. S. et al. (2014) Earth, Planets and Space 66:89.

[6] Loeffler M. J. et al. 2016. Meteoritics \& Planet. Sci., 51:261-275.

[7] Nakamura T. et al. (2011) Science 333:1113-1115.

[8] Noguchi T. et al. 2014. Meteoritics \& Planet. Sci., 49: 188-214.

[9] Bland P.A. et al. 2004. Meteoritics. \& Planet. Sci., 39:3-16.

[10]. Keller, L. P. et al. (2014) Earth, Planets and Space 66:71.

[11] Loeffler M. et al (2009) Icarus 195: 622-629.

[12] Matsumoto T. et al. (2019) Asteroid Science, Abstract \#2155. 